\title{
Efectos de un programa basado en el Análisis Conductual Aplicado en la enseñanza de Conceptos Éticos y Morales religiosos.
}

González-Gallo, Iván'; Ramírez, Diego Armando'; Villarreal, Carol Stefany; Parra-Bolaños, Nicolás 2(*)

'Universidad Autónoma de Bucaramanga, Programa de Psicología, Bucaramanga, Colombia

${ }^{2}$ Asociación Educar para el Desarrollo Humano, Laboratorio de Neurociencias y Educación, Buenos Aires, Argentina

\section{RESUMEN}

La educación es fundamental para el desarrollo pleno como seres humanos, puesto que es allí donde se aprenden conceptos básicos y parámetros de comportamiento para desenvolverse en el ámbito social. A lo largo de la historia han surgido diferentes modelos o paradigmas que buscan dar pautas, técnicas, herramientas y estrategias para cumplir los objetivos y metas propuestas en los procesos que se adelantan dentro de la educación y el diseño de los currículos. Uno de estos paradigmas es el planteado por el análisis de la conducta, el cual por su incorrecta e incompleta aplicación se ha tergiversado en su interpretación y ha recibido fuertes críticas en la práctica. Por lo anterior, esta investigación propone observar y establecer los efectos de la implementación de los principios del Análisis Conductual Aplicado en la enseñanza de valores éticos religiosos de 12 adolescentes de 11 a 13 años, pertenecientes al grupo de catequesis de la iglesia el Buen Pastor de Bucaramanga. Para esto se llevó a cabo una investigación con un diseño ABAB en donde se midieron las variables dependientes motivación y participación al aplicarse un programa de reforzadores sociales y economía de fichas. Los resultados evidenciaron que estas últimas estrategias del Análisis Conductual fueron efectivas para aumentar la participación y la motivación y el nivel de aprendizaje.

Palabras clave: tecnología de la educación; análisis conductual aplicado; economía de fichas.

\section{Effects of an Applied Behavioral Analysis based program in the teaching of Ethical and Moral Religious Concepts.}

\section{ABSTRACT}

Education is essential for integral development as human beings, due to learning of basic concepts and behavioral parameters occurring and developing in the social sphere. Throughout history, different models or paradigms have emerged seeking to give guidelines, techniques, tools and strategies to meet the objectives and goals proposed in processes carried out within the education and design of the study program. One of these paradigms is behaviorism, which due to its incorrect and incomplete application misrepresent the latter and has received strong criticism in practice. Therefore, this research proposes to observe and establish the effects of the application of the principles of Applied Behavioral Analysis in the teaching of religious ethical values of 12 adolescents aged 11 to 13 , belonging to the catechesis group of the Buen Pastor Church of Bucaramanga. In order to achieve this, an ABAB design research was carried out, measuring dependent variables motivation and participation after a social reinforcers and a token economy program was implemented. The results showed that these latest Behavioral Analysis strategies were effective in increasing participation, motivation and level of learning.

Keywords: educational technology; applied behavior analysis; token economy 


\section{INTRODUCCIÓN}

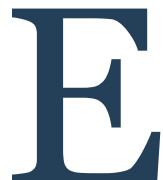

I paradigma conductual aplicado a la enseñanza de diversos tipos de conocimientos y/o habilidades ha tenido gran fuerza en los ámbitos científicos, de entrenamiento y educativo, puesto que se interesa en la relación existente entre la conducta de la persona y el ambiente en que se desenvuelve, basándose en unos principios que propician la modificación del último para la generación, aumento o disminución de conductas (Skinner-Vargas, 2009). La implementación de este paradigma en la educación se dificulta puesto que es visto como un modelo de educación unidireccional y reduccionista, críticas que han tergiversado los postulados reales planteados por este modelo, creando una idea mecanicista del mismo, que no permite explotar las ventajas que traen las estrategias conductuales (Pellón, 2013), dejando así de lado la oposición que Skinner (1966; 1975a), creador del conductismo radical, realizó a la educación tradicional y el uso del método hipotético-deductivo en la misma: Skinner no rechazó la existencia de propósitos, intenciones, deseos, intereses u objetivos, ni que éstos dirijan la conducta, lo que rechazó fue el recurso de utilizarlos como explicaciones últimas de la conducta, porque aún tales propósitos e intenciones tienen que ser explicados y sus orígenes siempre se encuentran en la historia de reforzamiento de la persona (Plazas, 2006, p.374).

B. F. Skinner propone el análisis experimental en su libro The Behavior of Organisms en 1938, base de lo que hoy se conoce como análisis conductual aplicado, el cual "está orientado a la conducta actual y a las variables que la mantienen en el presente, sin desconocer la historia previa o evolución de la conducta objeto de análisis", es decir, para la comprensión de la conducta se debe realizar un análisis funcional que muestre los estímulos que la anteceden, sus características (frecuencia, duración, fuerza o intensidad) y las consecuencias o postcedentes ambientales que le siguen inmediatamente (Rodas, 2009, p. 13; Skinner-Vargas, 2009). Por lo tanto, en el Análisis Experimental de la Conducta "el objeto de estudio lo constituyen las consecuencias medioambientales de la conducta operante; es decir, la conducta de los organismos se "esculpe y moldea" a partir de las consecuencias que éstas tienen sobre su medio" (Pulido \& Calzada, 2010, p. 297). Por esto se retoma lo siguiente. y a su vez son cambiados por las consecuencias de sus actos. Ciertos procesos, que los organismos humanos comparten con otras especies, alteran la conducta en forma tal que se logra un intercambio más seguro y útil con un medio ambiente en particular. Cuando se ha establecido la conducta apropiada, sus consecuencias funcionan a través de procesos similares para mantenerla en vigor. Si por azar el ambiente cambia, las formas antiguas de conducta desaparecen, y las nuevas consecuencias dan origen a formas nuevas (Skinner, 1953, p.11).

Por lo anterior se puede argumentar que Skinner estudió las interacciones del organismo y el ambiente, y la generación de consecuencias que mantienen esas interacciones en el tiempo, lo que acuñó con el término operante para distinguir el comportamiento que opera en el ambiente del comportamiento respondiente de Pavlov, ya que en este último el organismo se adapta al medio, pero no actúa sobre éste (Skinner-Vargas, 2009).

Así entonces, las relaciones entre el comportamiento de las personas y sus eventos externos se llaman contingencias. Dentro de estas últimas las que eliminan o reducen la ocurrencia de la conducta, está el castigo que tiene como función disminuir la frecuencia de una conducta - la fuerza de un comportamiento, ya sea añadiendo una consecuencia generalmente pero no exclusivamente aversiva para el organismo (castigo positivo) o disminuir la ocurrencia de la conducta suprimiendo una consecuencia que actúa como reforzador para el organismo (castigo negativo) (Skinner-Vargas, 2009; Rodas, 2009; Chance 2013). De acuerdo con la literatura, existen dos tipos de reforzadores, los positivos y los negativos. Los primeros incrementan la frecuencia de la respuesta al presentar un evento o postcedente para el organismo y los segundos incrementan la frecuencia de la respuesta por el retiro de una consecuencia, generalmente pero no exclusivamente aversiva para el organismo. Desde otro tipo de clasificación se plantean los reforzadores primarios, que presentan un origen más evolutivo y están asociados a necesidades fisiológicas, presentando un efecto de mayor magnitud ante condiciones de privación (p.e. comida, agua, algunas drogas, sexo, entre otros.); y los reforzadores secundarios, cuyo efecto depende de la posibilidad de ser intercambiados por reforzadores primarios (p.e. títulos académicos, halagos, objetos o pertenencias materiales, entre otros.) (Prada, 1995; Chance, 2019). 
Entre las técnicas operantes para potenciar y debilitar conductas se encuentran el contrato de contingencias, que consiste un acuerdo escrito entre dos o más personas que especifica claramente las conductas a ejecutar y las consecuencias en caso de realizarlas o no. También existe la economía de fichas (token economy), la cual consiste en entregar fichas al sujeto cuando realice o no una conducta, de modo que tales fichas puedan ser cambiadas por una variedad de reforzadores a manera de bienes, actividades o privilegios, que adquieren su valor dependiendo del individuo. En algunos diseños también se incluyen penalizaciones, como retirada de fichas contingentemente a la conducta indeseable y retirada de fichas contingentemente a la no emisión de la conducta que se quiere eliminar (Bados \& García, 2011).

Existen aplicaciones de dichos principios, categorías y técnicas del análisis experimental de la conducta en ambientes organizacionales, servicios de tratamiento y rehabilitación física y psicológica y resolución de problemas sociales. (Ayala, 1990; Robayo, 2010). Estos planteamientos también han sido llevados al campo educativo, y definen la enseñanza como la evidencia de que las acciones de una persona afectan lo que otra pueda hacer. (Pennington, Simacek, McComas, McMaster \& Elmquist, 2019; Sleeman, Friesen, Tyler-Merrick \& Walker, 2019) Además, dentro del desarrollo que el paradigma conductual ha tenido dentro del campo educativo se plantea que la enseñanza no solo es la pericia en un tema o en ciertos contenidos, sino que incluye el diseño de contingencias que mejoren el aprendizaje de los estudiantes y que "Ios estudiantes deben hacer algo más que sentarse y mirar para ser competentes en un campo. Por lo tanto, los educadores del comportamiento ponen la actividad del estudiante en el centro de los procedimientos de enseñanza" (Skinner-Vargas, 2009, p. 85).

En consecuencia, desde el punto de vista conductual, el proceso de enseñanza consiste en la organización adecuada de contingencias de reforzamiento con el objetivo de promover eficazmente el aprendizaje del alumno, en donde el maestro es el encargado del arreglo de dichas contingencias, creando un ambiente estimulante para el alumno que debe, a su vez, ser sujeto activo en el proceso de aprendizaje (Barrios, Resendiz \& Faro, 2012).

Así mismo, los valores éticos y morales religiosos hacen parte de la cultura donde se forman Revista Innovación Digital y Desarrollo Sostenible Junio 2020 Vol. 1 N. 1 las personas. Estos valores se han desarrollado a través de la historia de la humanidad, se han transmitido de generación en generación y dirigen la forma de pensar y actuar de determinada sociedad. Una manera de transmitir los valores éticos y morales religiosos ha sido la catequesis dada por la iglesia católica, que de una forma estructurada educa en estos aspectos a niños, jóvenes y adultos (Camarena \& Tunal, 2009). Por lo anterior, ésta se considera una forma de educación no formal, ya que traspasa el ámbito escolar, es sistemática y colabora a la transmisión de conocimientos culturales (Pastor, 1999).

La presente investigación buscó observar el efecto de las estrategias conductuales como mecanismos que ayudan a mejorar el aprendizaje, mediante el manejo de conductas en los educandos como la motivación y la participación enfocándose en la implementación del análisis conductual aplicado a la enseñanza de conceptos éticos y morales religiosos en un espacio de formación de catequesis.

\section{MÉTODO}

Este estudio consiste en un diseño de reversión A-B-A-B o diseño de retirada de cuatro fases, así pues, las fases $A$ son de línea base, es decir, en estas no se implementa el tratamiento y en las fases $B$, se manipulan las variables mediante el tratamiento. Este diseño tiene gran validez interna, además, como su última fase es de tratamiento, permite que los cambios en la conducta se evidencien en mayor medida y se mantengan (Bono y Arnau, 2014).

\section{PARTICIPANTES}

Para esta investigación se tomó una muestra de 12 adolescentes, entre los 11 y 13 años de edad, del barrio San Bernardo, ciudad de Floridablanca, departamento de Santander, Colombia, pertenecientes a un nivel socioeconómico categorizado como 2, que asistieron a un grupo de catequesis de primera comunión de la iglesia católica, género indiferente. Esta caracterización de los sujetos se muestra en la Tabla 1. 


\section{Caracterización de la muestra}

Variable

Frecuencia Porcentaje

Edad

$\begin{array}{ll}11 & 5 \\ 12 & 4 \\ 13 & 3\end{array}$

$0,44 \%$

$0,31 \%$

$0,25 \%$

Total

12

$100 \%$

Tabla 1 Caracterización de la muestra. Elaboración de los autores.

\section{Operacionalización de las conductas objetivo}

Motivación: traer las actividades de asimilación (actividades para la casa) y la Biblia, además de participar activamente en las actividades de la experiencia humana (actividad dinámica) y la lectura de la Biblia.

Participación: levantar la mano para hacer una pregunta $u$ ofrecer un aporte verbal referente al tema en curso.

Adquisición: expresar de forma escrita características del concepto tratado en la catequesis.

\section{INSTRUMENTOS}

Como técnica de recolección de datos se utilizará la observación, ya que es una técnica que consiste evidenciar atentamente el fenómeno, hecho o caso, tomar información y registrarla para su posterior análisis (Alberto \& Troutman, 2006).

Debido a las características de las conductas a observar, (características establecidas en la operacionalización de la conducta) se requiere de la observación y del registro de esta en formatos de tablas de frecuencia mostrados en las Figuras 1 y 2 , ya que estos formatos permiten consignar la ocurrencia de eventos discretos de cierta clase en un determinado tiempo. (Rodas, 2009) La dis-

Figura 1. Tabla de frecuencia para registrar la conducta de participación. Elaborada por los autores. Se registró / cuando ocurría la conducta.

\begin{tabular}{|l|l|l|l|l|}
\hline Nombre & Leyó & $\begin{array}{l}\text { Actividad de } \\
\text { asimilación }\end{array}$ & $\begin{array}{l}\text { Trajo } \\
\text { Biblia }\end{array}$ & $\begin{array}{l}\text { Participó en la } \\
\text { experiencia } \\
\text { humana }\end{array}$ \\
\hline & & & & \\
\hline
\end{tabular}

Figura 2. Tabla de registro de frecuencia para la conducta de motivación. Elaborada por los autores. Se registraba X: participó y O: no participó.

\section{PROCEDIMIENTO}

Los tutores legales de todos los participantes fueron debidamente informados sobre todos los aspectos de la investigación y firmaron un consentimiento escrito, así como los directivos de la parroquia, siguiendo las directrices éticas de la investigación en Colombia. Los participantes también fueron informados del procedimiento de la investigación y se contó con su aprobación. En relación con el diseño experimental de caso único $A B A B$, se diseñaron 7 sesiones donde se aplicaron reforzamientos de tipo social y tangible, los primeros en relación con el reconocimiento social y las felicitaciones, y los últimos con salir más temprano y obtener una ficha. En las dos primeras sesiones se estableció la línea base, en las siguientes 3 se aplicó el tratamiento, 15 días después se realizó otra sesión donde se hizo una reversión a la línea base con el fin de evaluar los efectos del cambio conductual en los adolescentes son debido a las variables de tratamiento. Finalmente, 
en la última sesión se aplicó de nuevo del tratamiento, para comprobar nuevamente cuales son los efectos en el cambio de conducta. Durante todas las etapas de éste se utilizó la técnica de observación, utilizando dos tipos de registro de frecuencia para los dos tipos de conducta que se estudiaron. Igualmente se realizó una evaluación breve sobre el valor trabajado en cada sesión, la cual se presenta a continuación:

\section{Primera sesión: Valorar el amor de la madre}

Segunda sesión: Valorar la vida

Tercera sesión: Trabajar en equipo

Cuarta sesión: El amor

Quinta sesión: Cuidar el medio ambiente

Sexta sesión: Apreciar la importancia de los

momentos importantes de la vida

Séptima sesión: Vivir en comunidad

El diseño de reversión $A B A B$ se utiliza para analizar la efectividad de la variable independiente sobre la dependiente e implica la aplicación secuencial de un tratamiento y el retiro de una intervención para verificar los efectos del tratamiento en el comportamiento. Al comparar repetidamente los datos de referencia (línea base) con los datos recopilados durante la aplicación de la estrategia de intervención, el investigador puede determinar si existe una relación funcional entre las variables dependientes e independientes (Alberto y Troutman, 2006). El diseño para esta investigación consta de las siguientes etapas:

Primera etapa, A (línea de base 1): la línea de base inicial, durante la cual se recopilaron datos sobre el comportamiento objetivo en las condiciones existentes antes de la introducción de la intervención. Se realizó en dos sesiones con el catequista a cargo para poder determinar la línea base por medio de los formatos de frecuencia, midiendo así la participación y la motivación de los participantes. Al final de cada una de estas sesiones se hizo una evaluación con una pregunta abierta sobre los valores trabajados, (aprecio por el amor de la madre y el significado de la vida humana, respectivamente). En la segunda sesión se hizo un encuadre del tratamiento con la población: en primer lugar se les indicó que a medida que se evidencie que en posteriores sesiones hicieran $y / o$ contestaran preguntas $u$ ofrecieran algún aporte importante referente al tema podrán salir 15 minutos antes, también se les planteó la realización de una actividad propuesta por ellos mismos contingente a la acumulación de Revista Innovación Digital y Desarrollo Sostenible Junio 2020 Vol. 1 N. 1 fichas, que fueron administradas por cada actividad de asimilación realizada (p.e. traer la Biblia, participar en la experiencia humana, por pasar a leer, entre otras.) teniendo como meta 130 fichas, lo cual se les recordó al inicio de las tres sesiones posteriores. Cabe destacar que pEfara cada evaluación del valor trabajado se elaboró un concepto criterio con el que se compararon las respuestas de los participantes.

Segunda etapa, B (intervención 1): la introducción inicial de la intervención seleccionada para alterar el comportamiento objetivo - realizada en tres sesiones-. Se realizó la aplicación del tratamiento, refuerzo social y mediante fichas, cada vez que los participantes manifestaron las conductas previamente operacionalizadas (motivación y participación). Los valores éticos y morales religiosos trabajados en las tres sesiones fueron: Compartir con los otros, Buscar la paz y el amor y Cuidar la naturaleza respectivamente.

Tercera etapa, A (línea de base 2): un retorno a las condiciones de línea de base originales retirando la intervención. Después de un término de 15 días se realizó una sesión de reversión del tratamiento, para la obtención de datos que permitan demostrar que el tratamiento es el que está mejorando la motivación y participación de la población. Estas actividades fueron evaluadas y los participantes no recibieron fichas ni refuerzo social en esta fase.

Cuarta etapa: B (intervención 2): reintroducción del procedimiento de intervención. Se volvió a aplicar el tratamiento para tener evaluar la confiabilidad en los resultados. En esta sesión se trabajaron los temas de Vivir en comunidad e Importancia del trabajo en equipo, aplicando el mismo procedimiento de la segunda etapa. Al final, se contaron, en presencia de todos los participantes, las fichas totales recogidas.

\section{RESULTADOS}

Los resultados obtenidos en cada sesión se presentan a continuación mostrando los efectos del tratamiento a lo largo de las sesiones. 


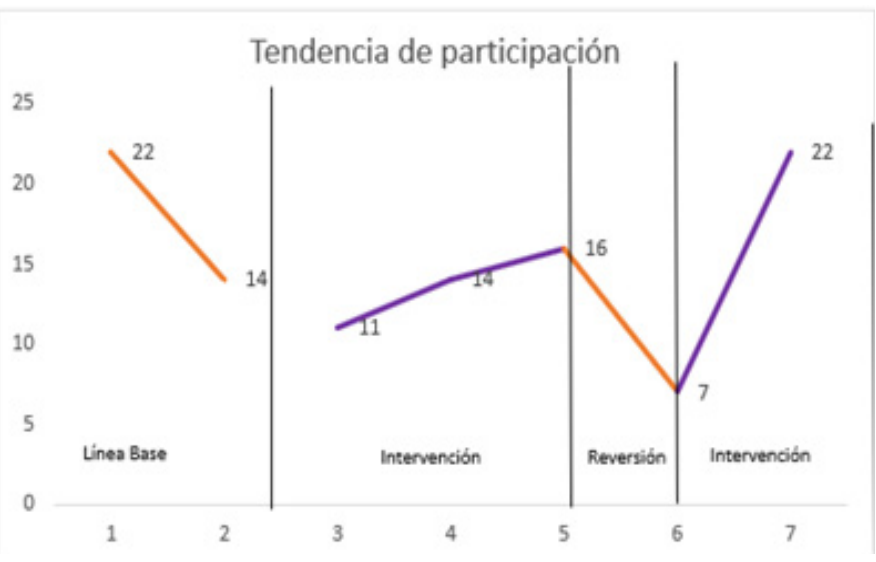

Figura 3. Tendencia de participación

En la Figura 3 se observan los resultados de la intervención $A B A B$ en la variable dependiente "Participación". Las variables independientes fueron reforzadores sociales tales como halagos, gestos de aprobación, reconocimiento público y frases como "Muy bien", "está excelente ese comentario" y "a eso era lo que quería llegar". También se utilizó en cada sesión de intervención una ficha o token como reforzador secundario, dentro de un programa de economía de fichas, a quien mostrara un mayor nivel de participación durante la sesión. En la sección de Línea Base se puede observar una participación inicial con frecuencia de $\mathrm{F}=22$ y un decrecimiento de esta en la siguiente evaluación, con una frecuencia de $\mathrm{F}=14$. Del mismo modo, la gráfica muestra una pendiente pronunciada negativa $(M=-8)$. Igualmente se puede evidenciar que en la primera intervención en la cual se aplica el tratamiento, hay una pendiente positiva $(M=2,5)$, lo cual muestra un aumento en el nivel de participación en comparación con la línea base. En la reversión se observa una pendiente negativa $(M=-9)$ Ilegando a un valor de frecuencia $(F=7)$ de participación inferior, incluso, al observado en la línea base realizada antes del tratamiento. Este resultado se constata con la nueva intervención, en la cual se observa una pendiente positiva $(M=15)$ llegando a una frecuencia $F=22$, la cual, supera los valores obtenidos durante las intervenciones de tratamiento anteriores.

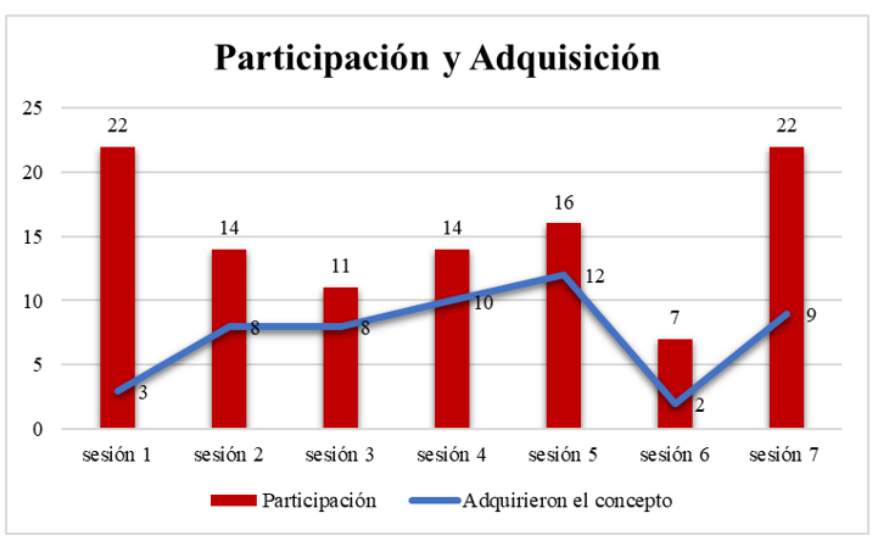

Figura 4. Tendencia de participación y adquisición del concepto enseñado.

En la Figura 4 se observa que hubo un aumento en la tasa de adquisición de conceptos éticos y morales religiosos en las sesiones de tratamiento, y esta decrece en la sesión de reversión. Es necesario denotar que en la primera sesión se observa por ejemplo que hay alta participación pero baja adquisición, mientras que el aumento de la tasa de participación y adquisición en la primera y segunda fase de intervención o tratamiento (tercera, cuarta, quinta y séptima sesión) se hace más coherente, ya que las pendientes presentan una proporcionalidad directa, en adelante en las sesiones 4 y 5 se presenta un aumento paralelo en la frecuencia entre participación ( $F=14$ a 16) y adquisición de conocimiento ( $F=10$ A 12). Cabe resaltar que, una vez retirado el tratamiento, el nivel de adquisición del concepto decreció considerablemente y posteriormente se produjo un aumento en la sesión donde se vuelve a aplicar el tratamiento. Igualmente, en esta última sesión se evidencia una participación $(\mathrm{F}=20)$ igual a la primera sesión de línea base, pero con la diferencia de que se dio una mayor tasa de adquisición del conocimiento por parte de la población.

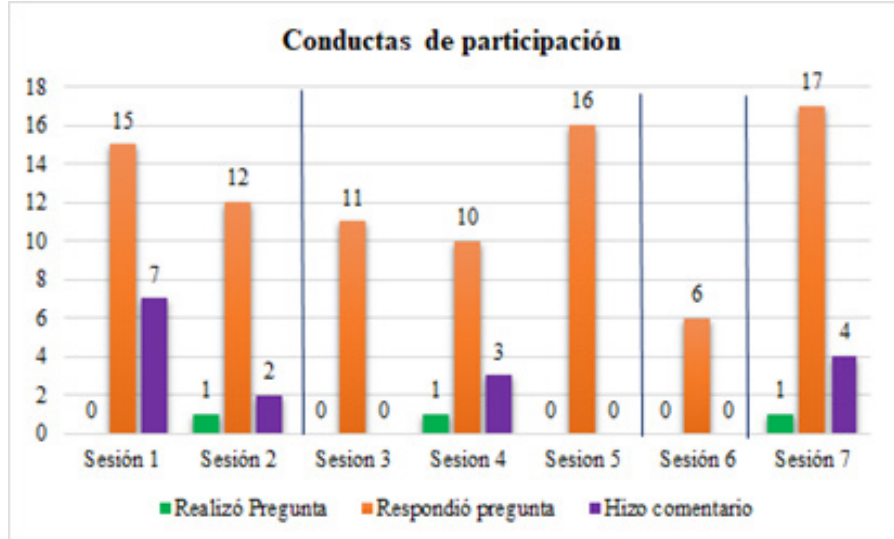

Figura 5. Conductas operacionalizadas como participación. 
En la Figura 5 se observa que las frecuencias de participación más altas se encuentran en la conducta de responder preguntas, puesto que las sesiones de catequesis fueron diseñadas para que se presentara este tipo de conducta en mayor medida. Referente a la conducta de realizar pregunta, siempre que se presentó, la frecuencia se mantuvo constante $(F=1)$. Por último, la conducta de hacer un comentario osciló entre valores de frecuencias de 2 a 7.

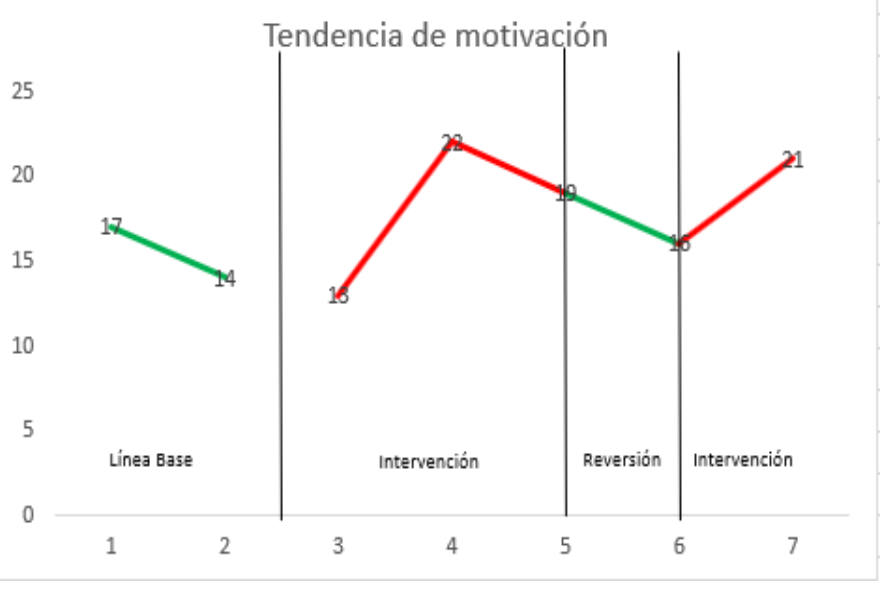

Figura 6. Tendencia de motivación

En la Figura 6 se observan los resultados de la intervención $A B A B$ en la variable dependiente "motivación", la cual se quiso aumentar en los participantes mediante un programa de economía de fichas propuesto por los investigadores. Así pues, las variables independientes fueron unos reforzadores secundarios tangibles, denominados fichas o token, que se obtenían por traer la Biblia, leerla, traer las actividades de asimilación del tema anterior y participar activamente en la actividad propuesta para catequesis. Dichos reforzadores serian canjeables por un reforzador de tipo recreacional, ya que al conseguir un acumulado de los mismos, los participantes serían premiados con una actividad que tuviera efecto reforzante en ellos. Por lo tanto, en la sesión de Línea Base se puede observar una motivación que va de una frecuencia de $F=17 \mathrm{~A} F=$ 14 existiendo así un decrecimiento de la misma, con una pendiente de $(\mathrm{M}=-3)$. Por otro lado, en las intervenciones de tratamiento se observa una pendiente positiva $(M=3)$ entre la sesión número 3 y 4, además de una frecuencia $F=19$ en la sesión 5 , lo cual muestra una tasa de motivación relativamente superior en comparación a la línea base. En la sesión de reversión se observa una pendiente negativa $(M=-3)$ haciéndose evidente un regreso a la línea base con una frecuencia de $\mathrm{F}=16$. Lo anterior muestra que la variable inde- pendiente tiene un efecto sobre la variable dependiente, que se constata en la sesión número 7 , donde se aplica de nuevo el tratamiento y se obtiene un aumento en la frecuencia de $F=21(M=5)$, puntuación cercana a la más alta obtenida en la primera fase de tratamiento, (sesión 4, F=22).

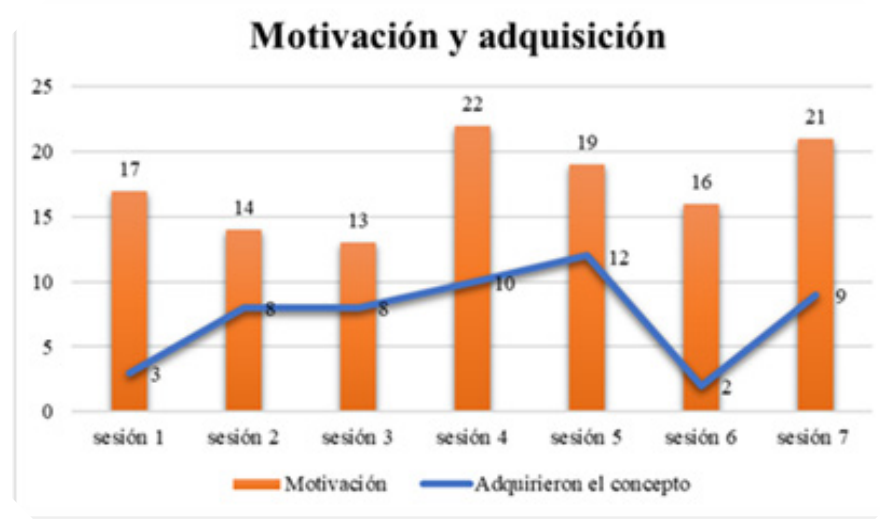

Figura 7. Tendencia de motivación y adquisición del concepto enseñado.

En la Figura 7 se observa que hubo un aumento en la tasa de adquisición de conceptos éticos y morales religiosos en las sesiones de intervención, y esta decrece en la sesión de reversión. Por esto, es de resaltar que en las sesiones donde no se reforzó las conductas operacionalizadas para motivación, hubo baja frecuencia de la misma en los participantes y un especial decrecimiento en la tasa de adquisición (aprendizaje) $(M=-10)$ con una frecuencia $F=2$ en la sesión número 6 , la cual corresponde a la sesión de retirada de tratamiento, llegando a elevarse la tasa de adquisición en la reintroducción del tratamiento hasta alcanzar a una frecuencia $\mathrm{F}=9$.

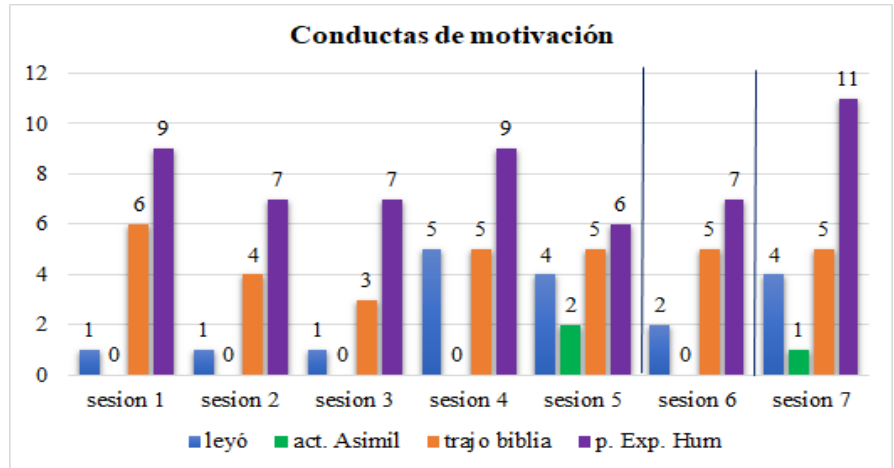

Figura 8. Conductas operacionalizadas como motivación.

En la Figura 8 se evidencia que aunque los valores de la línea base y los de las fases de tratamiento no distan mucho en número de frecuencia, hubo un cambio incremental en la variable dependiente en las fases de tratamiento sobre todo en la tarea de pasar a leer y un cambio en 
la baja frecuencia en la tarea de traer realizada la actividad de asimilación.

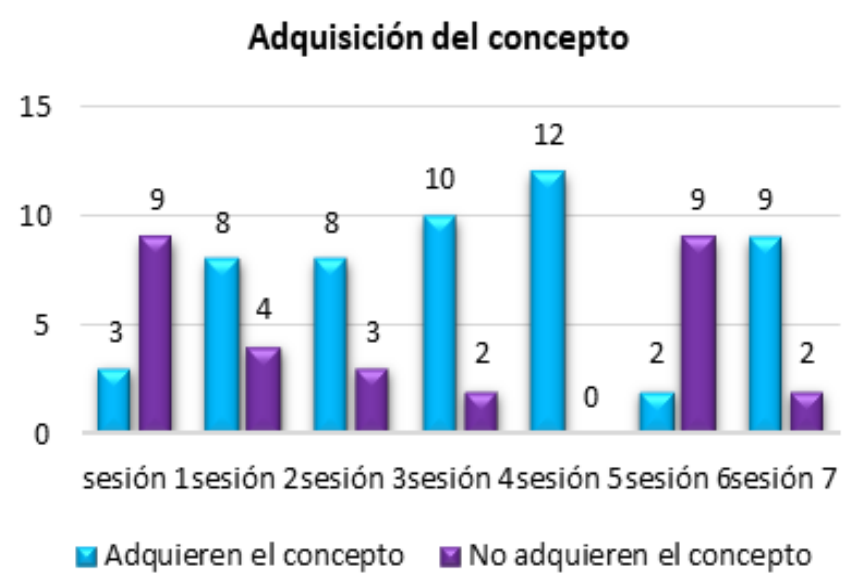

Figura 9. Frecuencias de adquisición del concepto en cada sesión.

En la Figura 9, en general se puede observar que el concepto enseñado en cada catequesis, tuvo, en la primera fase de las sesiones de tratamiento, un aumento de frecuencia en la cantidad de sujetos que adquirieron el concepto que va desde $F=8$ a $F=12$, con una pendiente de $M=2$ lo cual demuestra una mejoría. Se observa también con respecto a la línea base que el número de estudiantes que no adquirieron el concepto fue menor en la sesión de reversión que en la línea base $(F=3$ y $F=2)$. Sumado a esto, es relevante la séptima sesión en donde se vuelve a aplicar el tratamiento, confirmando que el cambio en la tasa de adquisición del concepto en la primera fase de tratamiento no está relacionado con variables ajenas a las independientes, ya que la variable dependiente llegó a una frecuencia de $F=9$ y una elevación de la pendiente de $\mathrm{M}=7$.

\section{DISCUSIÓN}

Los resultados permiten concluir que existe diferencia positiva en el nivel de aprendizaje de conceptos éticos y morales religiosos al desarrollar un programa de educación basado en el análisis conductual, por lo tanto, se acepta la hipótesis alternativa, ya que, aunque diferentes los efectos, el tratamiento los tuvo en ambas variables dependientes.

La variable participación fue operacionalizada basándose en definiciones que la consideran como una manera de ejercer un papel activo Revista Innovación Digital y Desarrollo Sostenible Junio 2020 Vol. 1 N. 1 por parte del estudiante manifestando sus inquietudes o aportando información relevante a la clase (Prieto, 2005). Los resultados muestran que hubo un aumento de la tasa de conductas de esta variable durante las sesiones de tratamiento y que en la sesión de reversión hubo un decremento de esta, la cual vuelve a aumentar en la sesión de reintroducción del tratamiento, lo que evidencia que la estrategia de aplicar refuerzos sociales tales como halagos y gestos de aprobación influyeron de forma positiva en la frecuencia y calidad de respuestas y aportes dados sobre el tema, ya que implicaron en los participantes una retroalimentación inmediata de aquellas conductas deseables planteadas inicialmente (Skinner-Vargas, 2009; Morell, 2009), identificándose así los efectos de las estrategias conductuales en la cantidad y calidad de participación en clase. Cabe resaltar que en la primera sesión de línea base la tasa de participación fue relativamente alta, lo cual puede explicarse por el hecho de que el primer día había una contingencia novedosa para los participantes (ver por primera vez al observador) (Domjan, 2007; Chance, 2013).

En cuanto a la variable de motivación se optó por reforzar mediante un programa de economía de fichas a una serie de actividades que se les dificultaba, en mayor medida, realizar a los participantes. Debido a que se considera la economía de fichas como un procedimiento efectivo para eliminar repertorios de conductas indeseables y con un valor motivacional en el aumento de la frecuencia de conductas deseables (González, 2005; Martinez, 2013), consiguiendo con este programa un cambio incremental positivo en algunas tareas de las conductas operacionalizadas como motivación utilizando un sistema de retroalimentación grupal e individual (Aguilar \& Navarro, 2008). Por otra parte, se encontró que la tasa de adquisición de los conceptos éticos y religiosos fue más alta en las sesiones de intervención comparada con las de línea base y reversión.

Lo anterior demuestra que la manipulación de las variables independientes tiene un efecto sobre la motivación y participación, que desde un enfoque conductual lleva a que el aprendizaje de dicho concepto se haga más visible en la población (Flores, 2015; Rueda, Mares, Gonzáles, Rivas \& Rocha, 2017). En dichas sesiones se observó mayor cantidad de sujetos que dieron una respuesta correcta hacia el significado del valor enseñado. No obstante, se evidencia en la figura 8 hay un incremento en la frecuencia de adquisición del concepto ético religioso que se en- 
señó en la segunda sesión de línea base, igual que en la primera sesión de tratamiento. Lo anterior puede deberse a que el tema de la sesión 2 fuera naturalmente interesante para los participantes. Además de esto, en dicha sesión se presentaron condiciones ambientales (no había más grupos de catequesis en el mismo piso, por ende, menos ruido) que permitieron la concentración del grupo en el tema.

En la primera sesión de tratamiento se observan frecuencias más bajas de motivación y participación en comparación a la línea base, esto se puede explicar porque hubo un lapso de tiempo más largo entre la segunda sesión de línea base y la primera sesión de tratamiento en comparación con las demás sesiones, debido a situaciones ajenas al control de los investigadores, y debido a lo anterior se pudo generar olvido en los participantes. Los resultados de la intervención muestran un aumento en el seguimiento de instrucciones para el desarrollo normal de la clase, aumento en la conducta pro-social y la participación voluntaria en clase, y una disminución de comportamientos agresivos, el conversar en clase y gritar, lo cual replica resultados de otras investigaciones hechas con métodos similares (Corsi, Barrera, Flores, Perivancich \& Guerra, 2009).

Las variables de motivación, participación y frecuencia de adquisición del concepto cambiaron con una pendiente positiva, demostrando el efecto en el aumento de conductas proactivas y productivas por parte de los participantes, los cuales tuvieron durante el transcurso del tratamiento la oportunidad de tomar la iniciativa en el momento de realizar las actividades planteadas, diseñadas con el fin de hacer que los participantes utilizaran herramientas de análisis, creatividad y dinamismo al momento de realizarlas, además se daban espacios donde de manera autónoma respondieran las preguntas y plantearan otras y se permitió que participaran en la elección de los reforzadores.

Con esto se evidencia cómo se logró a partir de los principios conductuales elaborar una acción estratégica pedagógica con una organización adecuada de contingencias de reforzamiento orientada a facilitar el aprendizaje. (González, 2005; Skinner-Vargas, 2009), en donde el maestro es el encargado del diseño e implementación de dichas contingencias, creando un ambiente estimulante sin dejar de lado el protagonismo de los educandos como principales actores de su proceso de aprendizaje, logrando que desempeñaran un papel autónomo, participativo y creativo, identif- icando consecuencias que refuerzan su conducta. Por lo tanto, estos resultados refutan una de las principales críticas a este modelo en el campo de la educación, en la que exponen que el estudiante es un ser pasivo que meramente recibe y réplica información (Inserra, 2016).

Teniendo en cuenta lo expuesto anteriormente se concluye que el uso de los principios del Análisis Conductual Aplicado en la enseñanza de valores éticos religiosos de en una muestra de adolescentes entre los 11 y 13 años, pertenecientes al grupo de catequesis de la iglesia el Buen Pastor de Bucaramanga, tuvo resultados que contribuyeron a su proceso de aprendizaje de manera positiva. Asimismo, al identificar el efecto de las estrategias conductuales en la cantidad y calidad de participación en clase y describir los cambios en la motivación de los adolescentes como resultado de la aplicación de estrategias conductuales se logra cumplir los objetivos que se establecieron para este estudio. Lo anterior sustenta uno de los planteamientos iniciales respecto a la funcionalidad de la aplicación de estrategias de enfoque conductual en el ámbito educativo.

Para finalizar, se recomienda realizar más investigación en este campo, bajo distintas contingencias (educación formal pública y privada, estudiantes de distintos niveles socioeconómicos, aplicación estable y contigua en el tiempo, mayor número de sesiones, diferentes tipos de reforzadores, control de distintas variables extrañas, entre otras). Es importante continuar aplicando estrategias que se rijan bajo principios básicos del aprendizaje, preparando a los educadores para la correcta aplicación de dichas estrategias, siendo esto fundamental para la efectividad de este tipo de intervenciones.

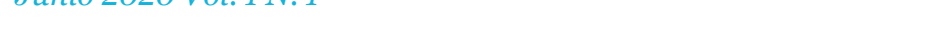




\section{REFERENCIAS}

Alberto, P. \& Troutman, A. (2006). Applied Behavior Analysis for Teachers, Seventh Edition. New Jersey: Pearson Education.

Ayala, H. (1990). La contribución de B.F Skinner a la psicología aplicada. Revista mexicana de análisis experimental de la conducta, 16(1) (2), 49-52.

Bados \& García (2011). Técnicas operantes. Departamento de Personalidad, Evaluación y Tratamiento Psicológicos, Facultad de Psicología. Universidad de Barcelona.

Barrios, J. G., Resendiz, M., \& Faro, T. (2012). Breve análisis del concepto de Educación Superior. Alternativas en psicología. 16(27), 34-41.

Bono, R. y Arnau, J. (2014). Diseños de caso único en ciencias sociales y de la salud. Madrid: Síntesis.

Camarena, M., \& Tunal, G (2009). La religión como una dimensión de la cultura. Nómadas. Critical Journal of Social and Juridical Sciences, 22 (2), 1-15.

Chance, P. (2013). Learning and behavior. Nelson Education.

Corsi, E., Barrera, p., Flores, C., Perivancich, X y Guerra, C. (2009). Efectos de un programa combinado de técnicas de modificación conductual para la disminución de la conducta disruptiva y el aumento de la conducta prosocial en escolares chilenos. Acta Colombiana de Psicología, 12 (1), 67-76.

Domjan, M. (2007). Principios de aprendizaje y conducta. Editorial Paraninfo.

Flores, O (2015). La participación de los estudiantes en el aula como factor determinante para mejorar la calidad de los aprendizajes (Tesis de maestría). Universidad Alberto Hurtado, Santiago, Chile.

González, A. (2005). Aportaciones de la psicología conductual a la educación. Sinéctica, Revista Electrónica de Educación, (25), 15-22.

Inserra, M (2016). Educación ideal: combinación del conductismo y constructivismo. Reflexión Pedagógica. Edición IV Ensayos de estudiantes de la Facultad de Diseño y Comunicación, (124), 79-83.

Martínez, S. (2013). El refuerzo positivo como estrategia motivacional en el aula de educación primaria. Propuesta didáctica (Tesis de pregrado). Universidad de Valladolid, Valladolid, España.

Morell, T. (2009). ¿Cómo podemos fomentar la participación en nuestras clases universitarias? Alicante: Editorial Marfil, S.A.

Pastor, M. (1999). Ámbitos de intervención en educación no formal. Una propuesta taxonómica. Ediciones Universidad de Salamanca, 11,183-215.
Pellón, R. (2013). Watson, Skinner y Algunas Disputas Dentro Del Conductismo. Revista Colombiana de Psicología, 22(2), 389-399.

Plazas, E. (2006). B. F. Skinner: la búsqueda de orden en la conducta voluntaria. Universitas Psychologica, 5(2), 371-384.

Pennington, B., Simacek, J., McComas, J., McMaster, K., \& Elmquist, M. (2019). Maintenance and generalization in functional behavior assessment/behavior intervention plan literature. Journal of Behavioral Education, 28(1), 27-53. https://doi.org/10.1007/ s10864-018-9299-6.

Prada, R. (1995). Escuelas psicológicas y psicoterapéuticas. Bogotá, San Pablo.

Prieto, M. (2005). La participación de los estudiantes: ¿Un camino hacia su emancipación? Theoria, 14 (1), 27-36

Pulido, M. \& Calzada, J. (2010). El Análisis Experimental de la Conducta: Algunos modelos experimentales de interés para las neurociencias. Revista Mexicana de Neurociencia, 11(4), 296-301.

Robayo, B. (2010). Sobre la relación entre el análisis de la conducta y los problemas de relevancia social. Revista Colombiana de Psicología, 19(1), 139-144.

Rodas, R. (2009). Análisis Conductual Aplicado. Manizales: Centro de publicaciones, Universidad de Manizales.

Rueda, E., Mares, G., Gonzáles, L., Rivas, O., \& Rocha, H. (2017). La participación en clase en alumnos universitarios: factores disposicionales y situacionales. Revista Iberoamericana de Educación Superior, 74(1), 149-162. https://doi.org/10.35362/ rie741632.

Rustom, A. (2012). Estadistica Descriptiva, Probabilidad e Inferencia: Una visión conceptual y aplicada. Santiago, Chile: Universidad de Chile.

Sleeman, M., Friesen, M., Tyler-Merrick, G., \& Walker, L. (2019). The Effects of Precision Teaching and Self-regulated Learning on Early Multiplication Fluency. Journal of Behavioral Education, 1-29. https:// doi.org/ 10.1007/s10864-019-09360-7.

Skinner, B. F. (1969). Contingencies of reinforcement. New York: Appleton-Century-Crofts.

Skinner, B. F. (1953). Science and human behavior. New York: Macmillan.

Skinner, B. F. (1975a). La conducta de los organismos: un análisis experimental. Barcelona: Fontanella. (Trabajo original publicado en 1938).

Skinner Vargas, J. (2009). Behavior Analysis for Effective Teaching. New York: Routledge. 